\title{
Inter-operator Dynamic Spectrum Sharing: A Stochastic Optimization Approach
}

\author{
S. K. Joshi, K. B. S. Manosha, M. Codreanu, and M. Latva-aho \\ Centre for Wireless Communications, University of Oulu, \\ \{sjoshi, shkapuru, codreanu, matti.latva-aho\}@ee.oulu.fi
}

\begin{abstract}
The problem of spectrum sharing between two operators in a dynamic network is considered. We allow both operators to share (a fraction of) their licensed spectrum band with each other by forming a common spectrum band. The objective is to maximize the gain in profits of both operators by sharing their licensed spectrum bands rather than using them exclusively, while considering the fairness among the operators. We use the notion of cooperative games, and model this problem as a two-person bargaining problem. The bargaining problem is cast as a stochastic optimization problem, which can be solved by using the elegant theory of Lyapunov optimization.
\end{abstract}

\section{INTRODUCTION}

In the traditional cellular systems, the radio spectrum is divided into a set of disjoint blocks which are assigned (licensed) to different operators on an exclusive basis. The assignment of exclusive spectrum bands to operators gives each operator the right to control their spectrum bands. However, when the entire spectrum band is considered, the exclusive allocation strategy often leads to a low spectrum utilization, because the operators may have different spectrum demands over the time and some part of the spectrum band can be underutilized [1], [2]. Therefore, spectrum sharing between the operators is required for better spectrum utilization, and to cope with the rapidly increasing spectrum demand [3], [4].

The operators can share their spectrum band with each other in two basic ways [5]: orthogonal sharing and non-orthogonal sharing. In the orthogonal sharing, operators are allowed to operate in each others spectrum bands; but at any time instance one spectrum band can be used only by one operator. Thus, the transmissions of the operators do not interfere with each other. In contrast, in the non-orthogonal sharing, operators are allowed to transmit on the same spectrum band at the same time and location. Here, the operators are required to coordinate their operation and choose transmission strategies to mitigate the inter-operator interference [6]. Inter-operator orthogonal spectrum sharing algorithms have been proposed in [7]-[10], and the non-orthogonal spectrum sharing algorithms are proposed in [11]-[13].

The key difference between the method introduced in this short paper and other existing works is that we are specifically taking into account the time-varying nature of the radio channel and address the problem of spectrum sharing in a dynamic network. To the best of our knowledge all existing

This research was supported by the Finnish Funding Agency for Technology and Innovation (Tekes), Academy of Finland, Broadcom, Nokia, Huawei, Anite, Elektrobit, and Nokia Foundation. spectrum sharing algorithms consider a static case (i.e., the spectrum sharing problem for a given instance). Thus, when these algorithms are applied over a period of time to a dynamic network, they may yield suboptimal performance and also may not ensure the stability of the network [14, Sec. 4.1]. As the spectrum sharing is a mutual agreement between operators to share their licensed bands over a period of time [3], it is important to consider the dynamics of a network (e.g., timevarying channels, dynamic traffic of the operators, etc.) in the problem formulation [15, Ch. 1].

We adopt the co-primary shared access model [3], and allow two operators to share (a fraction of) their licensed spectrum band with each other by forming a common spectrum pool. We share the common spectrum pool orthogonally between the operators. Both operators, with the unequal spectrum demands, need to be benefitted by sharing their licenses spectrum band with each other. Hence, we introduce a novel pricing rule in using the common spectrum pool for the co-primary shared access model [3]. Specifically, we allow the operators to use the spectrum up to the amount that they have contributed without any payment. But, if an operator uses more spectrum than it has contributed to the spectrum pool; then it has to pay to the other operator for the extra amount of spectrum it uses. Therefore, with this pricing rule an operator can maximize its profits either by using more spectrum band than actually it is licensed for, or leasing its spectrum band to the other operator who is in need.

Our goal is to maximize the gain in profits of both operators by sharing their licensed spectrum bands with each other, rather than using them exclusively. Moreover, we consider the fairness among the operators in the gain that they obtain by sharing their spectrum bands. Therefore, we use the notion of cooperative games, and model this problem as a two-person bargaining problem [16]-[20] ${ }^{1}$. Furthermore, the bargaining problem is cast as a stochastic optimization problem to consider the dynamics of the network [15, Ch. 1].

In this short paper we present a future research direction of our ongoing work on inter-operator spectrum sharing, which considers the dynamic of the network. We adopt a network utility maximization framework, and spectrum sharing between two operators is cast a cross-layer stochastic optimization problem [14], [15]. Here, we optimize the time average of the utilities of the operators, such that both operators fairly

\footnotetext{
${ }^{1}$ It is worth noting that bargaining problem leads to a fair solution, and proportional fairness [21] is a special case of it [18], [20].
} 
gain in there profits by sharing there spectrum with each other. The formulated problem can be solved by using the elegant theory of Lyapunov optimization [14], [15].

\section{System Model AND PROBlem Formulation}

We consider a wireless network consisting of a cell with two coexisting BSs, belonging to two different operators. The set of BSs is denoted by $\mathcal{N}$, and we label them with the integer values $n=1,2$. The transmission region of $\mathrm{BSs}$ is modeled as a disc with radius $R_{\mathrm{BS}}$ centered at the location of the BS. Each BS is equipped with $T$ transmit antennas, and each user is equipped with single receive antenna. We denote the set of all users in $n$th BS by $\mathcal{L}(n)$, and we label them with the integer values $l=1, \ldots, L_{n}$. Let each operator share equal ${ }^{2}$ amount of spectrum band $B \mathrm{~Hz}$ with the other operator. Hence, a total spectrum of bandwidth $2 B \mathrm{~Hz}$ is available for both operators. Furthermore, we assume that the total spectrum band $2 B \mathrm{~Hz}$ is split into $S$ subchannels. The set of subchannels is denoted by $\mathcal{S}$, and we label them with the integer values $s=1, \ldots, S$. Let the bandwidth of $s$ th subchannel is $w_{s} \mathrm{~Hz}$, and we assumed that it is smaller than a coherence bandwidth.

The network is assumed to be operating in slotted time with slots normalized to integer values $t \in\{1,2, \ldots\}$. At each time slot, a network controller partitions the $S$ subchannels between the operators (i.e., between the two BSs) ${ }^{3}$. Let the set of subchannels allocated to $n$th BS during time slot $t$ be $\mathcal{S}(n, t)$, and we label them with the integer values $s=1, \ldots, S_{n}(t)$. Hence, the signal received at $l$ th user of BS $n$ in subchannel $s$ during time slot $t$ can be expressed as

$$
\begin{aligned}
y_{n l, s}(t) & =d_{n l, s}(t) \mathbf{h}_{n l, s}^{\mathrm{H}}(t) \mathbf{m}_{n l, s}(t) \\
& +\sum_{j \in \mathcal{L}(n), j \neq l} d_{n j, s}(t) \mathbf{h}_{n l, s}^{\mathrm{H}}(t) \mathbf{m}_{n j, s}(t)+n_{n l, s}(t),
\end{aligned}
$$

where $d_{n l, s}(t) \in \mathbb{C}$ represents information symbol associated to $l$ th user of BS $n$ in subchannel $s, \mathbf{h}_{n l, s}^{\mathrm{H}}(t) \in \mathbb{C}^{1 \times T}$ is the channel matrix from $n$th $\mathrm{BS}$ to its $l$ th user in subchannel $s, \mathbf{m}_{n l, s}(t) \in \mathbb{C}^{T}$ is the transmit beamformer associated to $l$ th user of BS $n$ in subchannel $s$, and $n_{n l, s}(t)$ is circular symmetric complex Gaussian noise with power spectral density $N_{0}$. We assume that $d_{n l}(t)$ is normalized such that $\mathrm{E}\left|d_{n l, s}(t)\right|^{2}=1$. Furthermore, we assume that data streams are independent, i.e., $\mathrm{E}\left\{d_{n l, s}(t) d_{n j, s}^{*}(t)\right\}=0$ for $l \neq j$, where $l, j \in \mathcal{L}(n)$ and $n \in \mathcal{N}$.

In this paper, we consider the case where all receivers are using single-user detection (i.e., a receiver decodes its intended signal by treating all other interfering signals as noise), and assume that the achievable rate of $l$ th user of $n$th BS during time slot $t$ is given by [22, Ch. 5]

$$
\begin{aligned}
& r_{n l}(t) \triangleq r_{n l}\left(\mathcal{S}(n, t), \mathbf{m}_{n}(t)\right)= \\
& \sum_{s \in \mathcal{S}(n, t)} w_{s} \log _{2}\left(1+\frac{\left|\mathbf{h}_{n l, s}^{\mathrm{H}}(t) \mathbf{m}_{n l, s}(t)\right|^{2}}{N_{0} w_{s}+\sum_{j \in \mathcal{L}(n), j \neq l}\left|\mathbf{h}_{n l, s}^{\mathrm{H}}(t) \mathbf{m}_{n j, s}(t)\right|^{2}}\right)
\end{aligned}
$$

\footnotetext{
${ }^{2}$ The work can be easily generalized to the case where operators share different portions of the spectrum bands with each other.

${ }^{3}$ We use the terminologies BS and operator interchangeably
}

where we use the notation $\mathbf{m}_{n}(t)$ to denote a vector obtained by stacking $\mathbf{m}_{n l, s}(t)$ for all $l \in \mathcal{L}(n)$ and $s \in \mathcal{S}(n, t)$ on top of each other, i.e., $\mathbf{m}_{n}(t)=$ $\left[\mathbf{m}_{n 1,1}(t)^{\mathrm{T}}, \ldots, \mathbf{m}_{n L_{n}, S_{n}(t)}(t)^{\mathrm{T}}\right]^{\mathrm{T}}$. Furthermore, we assume that the power allocation is subject to a maximum power constraint $\sum_{l \in \mathcal{L}(n)} \sum_{s \in \mathcal{S}(n, t)}\left\|\mathbf{m}_{n l, s}(t)\right\|_{2}^{2} \leq p_{n}^{\max }$ for each BS $n \in \mathcal{N}$.

\section{A. Spectrum pricing}

At each time slot, the common spectrum pool $2 B \mathrm{~Hz}$ (i.e., the set of $S$ subchannels) is partitioned between the operators. The total spectrum band allocated to operator $n \in \mathcal{N}$ during time slot $t$ is $\sum_{s \in \mathcal{S}(n, t)} w_{s}$. We assume that both operators can use up to the amount of spectrum that they put in the spectrum pool without any payment. But, the operator pays for an extra band of spectrum, if it uses more spectrum than it has put in the common spectrum pool, to the other operator. Specifically, if spectrum band used by $n$th operator $\sum_{s \in \mathcal{S}(n, t)} w_{s}$ is more than $B \mathrm{~Hz}$, operator $n$ pays to the other operator (i.e., opponent of $n$th operator) for the extra band of spectrum $\left(\sum_{s \in \mathcal{S}(n, t)} w_{s}\right.$ $B) \mathrm{Hz}$. The amount to be paid is determined by the pricing rule established by the operators.

Let $q_{n}(t)$ be the per-unit price of spectrum during time slot $t$ set by $n$th operator to charge its opponent for using the extra spectrum band. To simplify the notation, let us use $\underline{n}$ to denote the opponent of $n$th operator ${ }^{4}$. Then the payment from operator $n \in \mathcal{N}$, for using the extra extra band of spectrum, to its opponent is $q_{\underline{n}}(t)\left(\sum_{s \in \mathcal{S}(n, t)} w_{s}-B\right)^{+}$.

\section{B. Network Queuing and Time Average Profit}

We consider a network utility maximization (NUM) framework similar to the one considered in [14, Sec. 5.1], [15, Ch. 5]. Specifically, exogenously arriving data is not immediately admitted to the network layer ${ }^{5}$. Instead, the exogenous data is first placed in the transport layer storage reservoirs. Then at each time slot a flow control decision is made, and decides the amount of each user data to be admitted to the network layer. Let $a_{n l}(t)$ denote the amount of data of $l$ th user of $n$th BS admitted in a network layer of BS $n$ during time slot $t$. At the network layer, each BS maintains a set of internal queues for storing current backlog (or unfinished work) of its users. Let $Q_{n l}(t)$ represents the current backlog of $l$ th user in $n$th BS. Then the evolution of the size of $Q_{n l}(t)$ is given by [14]

$$
Q_{n l}(t+1)=\max \left[Q_{n l}(t)-r_{n l}(t), 0\right]+a_{n l}(t),
$$

for all $n \in \mathcal{N}$ and $l \in \mathcal{L}(n)$, where $r_{n l}(t)$ is the transmission rate (defined in (2)) offered to $l$ th user of $n$th BS during time slot $t$. Here, we adopt the notion of strong stability ${ }^{6}$, and we say that the network is strongly stable if

$\bar{Q}_{n l} \triangleq \limsup _{t \rightarrow \infty} \frac{1}{t} \sum_{\tau=1}^{t} \mathrm{E}\left\{Q_{n l}(\tau)\right\}<\infty, n \in \mathcal{N}, l \in \mathcal{L}(n)$,

${ }^{4}$ For operator $n=1$, its opponent is $\underline{n}=2$. Similarly, for operator $n=2$, its opponent is $\underline{n}=1$.

${ }^{5} \mathrm{We}$ assume that the arrival data rate is outside the network capacity region. In a case, if a arrival data rate is within the network capacity region, it can be treated via a techniques of $[14$, Sec. 4$]$

${ }^{6} \mathrm{~A}$ definition of strong stability is general, and it also implies other forms of stability [15, Th. 2.8]. 
where the expectation depends on the control policy, and is with respect to the random channel states and the control actions made in reaction to these channel states. Intuitively, expression (4) means that a queue is strongly stable if its time average backlog is finite; and a network is strongly stable if all individual queues of the network are strongly stable.

At each time slot, for $l$ th user of $n$th BS the network controller admits $a_{n l}(t)$ data into the internal queue for transmission. Note that under network stability, admitted data $a_{n l}(t)$ for all $t$ in the internal queue is transmitted to the corresponding user over a finite period of time [15]. Thus, we define an utility of the user in terms of admitted data rate $a_{n l}(t)$, instead of transmission rate $r_{n l}(t)$. To define the utility of $l$ th user of BS $n$, let $\bar{a}_{n l}(t)$ denotes the admitted time average rate up to time slot $t$, i.e., $\bar{a}_{n l}(t) \triangleq \frac{1}{t} \sum_{\tau=1}^{t} \mathrm{E}\left\{a_{n l}(\tau)\right\}$. Then, associated with each user, we define a non-decreasing concave utility function $g_{n l}\left(\bar{a}_{n l}(t)\right)$. The utility function $g_{n l}\left(\bar{a}_{n l}(t)\right)$ represents a monetary measure of the satisfaction that $n$th operator receives by sending data to its $l$ th user based on its current data rate $\bar{a}_{n l}(t)$. Finally, we define the time average expected profit of $n$th operator as

$$
\begin{aligned}
\bar{U}_{n} \triangleq & \liminf _{t \rightarrow \infty}\left(\sum_{l \in \mathcal{L}(n)} g_{n l}\left(\bar{a}_{n l}(t)\right)\right. \\
& +\frac{1}{t} \sum_{\tau=1}^{t} \mathrm{E}\left\{q_{n}(\tau)\left(\sum_{s \in \mathcal{S}(\underline{n}, \tau)} w_{s}-B\right)^{+}\right\} \\
& \left.-\frac{1}{t} \sum_{\tau=1}^{t} \mathrm{E}\left\{q_{\underline{n}}(\tau)\left(\sum_{s \in \mathcal{S}(n, \tau)} w_{s}-B\right)^{+}\right\}\right),
\end{aligned}
$$

where the second right hand term in (5) represents an amount that $n$th operator gets by leasing its spectrum band to its opponent; and the third right hand term represents an amount that $n$th operator pays to its opponent for renting the extra spectrum band. Note that during any given time slot, only one operator can use more than $B \mathrm{~Hz}$ from the spectrum pool $2 B$ Hz. Hence, during any given time slot, operators either lease or rent a portion of the common spectrum band. Specifically, during time slot $\tau$, either term $q_{n}(\tau)\left(\sum_{s \in \mathcal{S}(n, \tau)} w_{s}-B\right)^{+}$or term $q_{\underline{n}}(\tau)\left(\sum_{s \in \mathcal{S}(n, \tau)} w_{s}-B\right)^{+}$is nonzero.

\section{Problem Formulation}

Our objective is to maximize the gain in profits of both operators by sharing their licensed spectrum bands with each other, rather than using them exclusively. Furthermore, we consider the fairness among the operators in the gain that they obtain. To do this, we model the spectrum sharing between two operators as a two-person bargaining problem [16]-[19] and cast as a stochastic optimization problem.

Let $U_{n}^{0}$ denotes the utility gain of $n$th operator that it gets before sharing its spectrum band with the other operator. In the context of bargaining problem, the utility $U_{n}^{0}$ is commonly known as a disagreement point, and it is assumed to be known. We assume that each operator knows a value of $U_{n}^{0}$ with their past experience. Then the benefits of the operators obtained by sharing their license spectrum bands with each other is $\bar{U}_{n}-U_{n}^{0}$ for all $n \in \mathcal{N}$. For tractability, we assume that a per-unit price of the spectrum band set by each operator is bounded, i.e., $0 \leq q_{n}(t) \leq q^{\max }$ for all $n \in \mathcal{N}$. Then the optimization problem to maximize the gain in operators profits, fairly $^{7}{ }^{8}$, subject to the network stability and the maximum power constraint for each BS can be expressed as

$$
\begin{aligned}
\operatorname{maximize} & \sum_{n \in \mathcal{N}} \log \left(\bar{U}_{n}-U_{n}^{0}\right) \\
\text { subject to } & \bar{Q}_{n l}<\infty, \quad n \in \mathcal{N}, l \in \mathcal{L}(n) \\
& 0 \leq q_{n}(t) \leq q^{\max }, \quad n \in \mathcal{N}, \forall t \\
& \sum_{l \in \mathcal{L}(n)} \sum_{s \in \mathcal{S}(n, t)}\left\|\mathbf{m}_{n l, s}(t)\right\|_{2}^{2} \leq p_{n}^{\max }, n \in \mathcal{N}, \forall t \\
& \mathcal{S}(1, t) \cap \mathcal{S}(2, t)=\emptyset, \quad \mathcal{S}(1, t), \mathcal{S}(2, t) \subseteq \mathcal{S}, \forall t,
\end{aligned}
$$

with variables $\left\{q_{n}(t), \mathcal{S}(n, t)\right\}_{n \in \mathcal{N}},\left\{a_{n l}(t)\right\}_{n \in \mathcal{N}, l \in \mathcal{L}(n)}$, and $\left\{\mathbf{m}_{n l, s}(t)\right\}_{n \in \mathcal{N}, l \in \mathcal{L}(n), s \in \mathcal{S}}$ for all $t \in\{1,2, \ldots\}$; where $\bar{U}_{n}$ and $\bar{Q}_{n l}$ are defined in (5) and (4), respectively. The constraint (6a) ensures that the network is stable. The constraint (6c) limits the total transmit power of each BS, and constraint (6d) ensures that a subchannel is allocated only to a single operator.

\section{DynAmiC Algorithm Via LyAPUnOV OptimizATION}

In this section we use the Lyapunov optimization technique [14], [15] to solve problem (6). To do this, first, we equivalently reformulate problem (6) by introducing auxiliary variables $\mu_{n}(t)$ for all $n \in \mathcal{N}$ and time slot $t$ as [14, Sec. 6.2]

$$
\begin{array}{ll}
\operatorname{maximize} & \sum_{n \in \mathcal{N}} \log \left(\bar{\mu}_{n}\right) \\
\text { subject to } & \bar{\mu}_{n} \leq \bar{U}_{n}-U_{n}^{0}, \quad n \in \mathcal{N} \\
& \text { constraints }(6 a)-(6 d),
\end{array}
$$

with variables $\left\{\mu_{n}(t), q_{n}(t), \mathcal{S}(n, t), a_{n l}(t)\right\}_{n \in \mathcal{N}, l \in \mathcal{L}(n)}$, and $\left\{\mathbf{m}_{n l, s}(t)\right\}_{n \in \mathcal{N}, l \in \mathcal{L}(n), s \in \mathcal{S}}$ for all $t \in\{1,2, \ldots\}$; where $\bar{\mu}_{n}$ is a time average of variable $\mu_{n}(t)$. Then, constrain (7a) is enforced by transforming it into a queue stability problem [14]. Let $\left\{X_{n}(t)\right\}_{n \in \mathcal{N}}$ be virtual queues associated with constraint (7a), and we update it at each time slot as

$$
X_{n}(t+1)=\max \left[X_{n}(t)-x_{n}^{\text {out }}(t), 0\right]+x_{n}^{\text {in }}(t),
$$

where

$$
\begin{gathered}
x_{n}^{\mathrm{out}}(t)=\sum_{l \in \mathcal{L}(n)} g_{n l}\left(a_{n l}(t)\right)+q_{n}(t)\left(\sum_{s \in \mathcal{S}(\underline{n}, t)} w_{s}-B\right)^{+}, \\
x_{n}^{\mathrm{in}}(t)=\mu_{n}(t)+U_{n}^{0}+q_{\underline{n}}(t)\left(\sum_{s \in \mathcal{S}(n, t)} w_{s}-B\right)^{+} .
\end{gathered}
$$

Now we summarize the steps of the proposed dynamic control algorithm based on Lyapunov optimization technique [14], [15] to solve problem (6) in Algorithm 1. A detailed derivation of the algorithm is omitted here due to the space limitations.

\footnotetext{
${ }^{7}$ For $U_{n}^{0}=0$ for all $n \in \mathcal{N}$, problem (6) is a proportional fair utility maximization problem [18], [21], [15, Ch. 5]. Thus, the objective function of problem (6) is a generalized proportional fairness objective [20], [23].

${ }^{8}$ Efficient utilization of the common spectrum pool can be obtained by maximizing the social welfare objective $\sum_{n \in \mathcal{N}} \bar{U}_{n}$, without regards to the spectrum prices $\left\{q_{n}\right\}_{n \in \mathcal{N}}$ because the payment will be canceled out. However, the maximization of the social welfare objective may not ensure the fairness in operator profits. In co-primary spectrum access, both operators want to maximize their profit, as both operators put their licensed spectrum band in the common spectrum pool. In other word, a fair distribution in the operator's profit is desirable.
} 
Algorithm 1: dynamic control algorithm.

1) Pricing: for each $n \in \mathcal{N}$, set $q_{n}(t)$ as

$$
q_{n}(t)= \begin{cases}q^{\max } & \text { if } X_{n}(t)>X_{\underline{n}}(t) \\ 0 & \text { otherwise. }\end{cases}
$$

2) Flow control: for each $n \in \mathcal{N}$, flow rate $a_{n l}(t)=a_{n l}$ for all $l \in \mathcal{L}(n)$, where $\left\{a_{n l}\right\}_{l \in \mathcal{L}(n)}$ solves the problem

$$
\begin{array}{ll}
\text { maximize } & X_{n}(t) \sum_{l \in \mathcal{L}(n)} g_{n l}\left(a_{n l}\right)-\sum_{l \in \mathcal{L}(n)} Q_{n l}(t) a_{n l} \\
\text { subject to } & 0 \leq a_{n l} \leq A^{\max }, \quad l \in \mathcal{L}(n),
\end{array}
$$

with variables $\left\{a_{n l}\right\}_{l \in \mathcal{L}(n)}$, where $A^{\max }>0$ is the algorithm parameter as described in [14, Sec. 4.2.1].

3) Auxiliary variable: for each $n \in \mathcal{N}$, auxiliary variable $\mu_{n}(t)=\mu_{n}$, where $\mu_{n}$ solves the problem

$$
\begin{array}{ll}
\text { maximize } & V \log \left(\mu_{n}\right)-X_{n}(t) \mu_{n} \\
\text { subject to } & 0 \leq \mu_{n} \leq \mu^{\max },
\end{array}
$$

with variables $\mu_{n}$, where $V>0$ and $\mu^{\max }>0$ is the algorithm parameter as described in [15, Ch. 5] .

4) Resource allocation:

$$
\begin{array}{cl}
\text { maximize } & \sum_{n \in \mathcal{N}} \sum_{l \in \mathcal{L}(n)} Q_{n l}(t) r_{n l}\left(\mathcal{S}_{n}, \mathbf{m}_{n}\right) \\
& +\sum_{n \in \mathcal{N}} X_{n}(t) q_{n}(t)\left(\sum_{s \in \mathcal{S}(\underline{n})} w_{s}-B\right)^{+} \\
& -\sum_{n \in \mathcal{N}} X_{n}(t) q_{\underline{n}}(t)\left(\sum_{s \in \mathcal{S}(n)} w_{s}-B\right)^{+} \\
\text {subject to } & \sum_{l \in \mathcal{L}(n)} \sum_{s \in \mathcal{S}(n)}\left\|\mathbf{m}_{n l, s}\right\|_{2}^{2} \leq p_{n}^{\max }, \quad n \in \mathcal{N} \\
& \mathcal{S}(1) \cap \mathcal{S}(2)=\emptyset, \quad \mathcal{S}(1), \mathcal{S}(2) \subseteq \mathcal{S},
\end{array}
$$

with variables $\left\{\mathbf{m}_{n l, s}\right\}_{n \in \mathcal{N}, l \in \mathcal{L}(n), s \in \mathcal{S}}$ and $\{\mathcal{S}(n)\}_{n \in \mathcal{N}}$. Set $\mathbf{m}_{n l, s}(t)=\mathbf{m}_{n l, s}$ and $\mathcal{S}(n, t)=\mathcal{S}(n)$ for all $n \in$ $\mathcal{N}, l \in \mathcal{L}(n)$, and $s \in \mathcal{S}(n)$.

5) Queue update: update $\left\{Q_{n l}(t+1)\right\}_{n \in \mathcal{N}, l \in \mathcal{L}(n)}$ and $\left\{X_{n}(t+1)\right\}_{n \in \mathcal{N}}$ by using expressions (3) and (8), respectively. Set $t=t+1$ and go to step 1 .

Observe that except step 4, the problems in each step of Algorithm 1 are decoupled into $N$ subproblems, one for each operator. Thus, by solving step 4 in centralized and distributed manner, we can obtain both centralized and distributed versions of Algorithm 1. By using Theorem [14, Th. 5.4], we can show that the objective value of problem (6) obtained by running Algorithm 1 is within $O(1 / V)$ of the optimal value, with a tradeoff in average queue backlog that is $O(V)$.

\section{CONCLUSION}

In this paper we suggest a novel approach for inter-operator spectrum sharing, specifically design to operate in a dynamic network environment. We have allowed operators to share their licensed spectrum bands with each other by forming a common spectrum band. Two-person bargaining framework has been used to model the spectrum sharing problem, and we have cast it as a stochastic optimization problem. The dynamic control algorithm is derived to solve this problem by using the elegant theory of Lyapunov optimization. A detailed description of the solution method is omitted here due to the space limitations but we refer the interested reader to [24] for a detailed derivation.

\section{REFERENCES}

[1] FCC, "Notice for proposed rulemaking: Facilitating opportunities for flexible, efficient, and reliable spectrum use employing cognitive radio technologies," ET Docket No. 03-108, Dec. 2003.

[2] I. Akyildiz, W.-Y. Lee, M. Vuran, and S. Mohanty, "A survey on spectrum management in cognitive radio networks," IEEE Commun. Mag., vol. 46, no. 4, pp. 40-48, Apr. 2008.

[3] Nokia Solutions and Networks Oy, "Optimising spectrum utilisation towards 2020," 2014. [Online]. Available: http://networks.nokia.com/ file/30301/optimising-spectrum-utilisation-towards-2020

[4] E. A. Jorswieck and et al., "Resource sharing in wireless networks: The SAPHYRE approach," in Proc. Future Network Mobile Summit, Jun. 2010, pp. 1-8.

[5] E. A. Jorswieck, L. Badia, T. Fahldieck, E. Karipidis, and J. Luo, "Spectrum sharing improves the network efficiency for cellular operators," IEEE Commun. Mag., vol. 52, no. 3, pp. 129-136, Mar. 2014.

[6] J. Lindblom and E. G. Larsson, "Does non-orthogonal spectrum sharing in the same cell improve the sum-rate of wireless operators?" in Proc. IEEE Works. on Sign. Proc. Adv. in Wirel. Comms., Jun. 2012, pp. 6-10.

[7] M. Bennis and J. Lilleberg, "Inter base station resource sharing and improving the overall efficiency of b3g systems," in Proc. IEEE Veh. Technol. Conf., Sep. 2007, pp. 1494-1498.

[8] H. Kamal, M. Coupechoux, and P. Godlewski, "Inter-operator spectrum sharing for cellular networks using game theory," in Proc. IEEE Int. Symp. Pers., Indoor, Mobile Radio Commun., Sep. 2009, pp. 425-429.

[9] L. Anchora, L. Badia, E. Karipidis, and M. Zorzi, "Capacity gains due to orthogonal spectrum sharing in multi-operator LTE cellular networks," in Proc. Int. Symp. Wireless Commun. Systems, Aug. 2012, pp. 286-290.

[10] P. Luoto, P. Pirinen, M. Bennis, S. Samarakoon, S. Scott, and M. Latvaaho, "Co-primary multi-operator resource sharing for small cell networks," IEEE Trans. Wireless Commun., vol. 14, no. 6, pp. 3120-3130, June 2015.

[11] E. Karipidis, D. Gesbert, M. Haardt, K.-M. Ho, E. Jorswieck, E. Larsson, J. Li, J. Lindblom, C. Scheunert, M. Schubert, and N. Vucic, "Transmit beamforming for inter-operator spectrum sharing," in Proc. Fut. Net. Mob. Summit, Jun. 2011, pp. 1-8.

[12] E. G. Larsson and E. A. Jorswieck, "Competition versus cooperation on the MISO interference channel," IEEE J. Select. Areas Commun., vol. 26, no. 7, pp. 1059-1069, Sep. 2008.

[13] J. Luo, J. Lindblom, J. Li, R. Mochaoura, A. Kortke, E. Karipidis, M. Haardt, E. Jorswieck, and E. G. Larsson, "Transmit beamforming for inter-operator spectrum sharing: From theory to practice," in Proc. Int. Symp. Wireless Commun. Systems, Aug. 2012, pp. 291-295.

[14] L. Georgiadis, M. J. Neely, and L. Tassiulas, "Resource allocation and cross-layer control in wireless networks," Found. Trends Net., vol. 1, no. 1, pp. 1-144, Apr. 2006.

[15] M. J. Neely, Stochastic Network Optimization with Application to Communication and Queueing Systems, ser. Synthesis Lectures on Communication Networks. San Rafael, CA: Morgan \& Claypool, 2010, vol. 7.

[16] L. Gao, G. Iosifidis, J. Huang, L. Tassiulas, and D. Li, "Bargainingbased mobile data offloading," IEEE J. Select. Areas Commun., vol. 32, no. 6, pp. 1114-1125, Jun. 2014.

[17] J. Nash, "The bargaining problem," Econometrica, vol. 18, no. 2, pp. 155-162, 1950.

[18] H. Boche and M. Schubert, "A generalization of nash bargaining and proportional fairness to log-convex utility sets with power constraints," IEEE Trans. Inform. Theory, vol. 57, no. 6, pp. 3390-3404, Jun. 2011.

[19] V. V. Vazirani, "The notion of a rational convex program, and an algorithm for the arrow-debreu nash bargaining game," in Pro. ACMSIAM Symp. Discrete Algorithms, 2012, pp. 973-992.

[20] Z. Han, Z. Ji, and K. Liu, "Fair multiuser channel allocation for OFDMA networks using nash bargaining solutions and coalitions," IEEE Trans. Commun., vol. 53, no. 8, pp. 1366-1376, Aug. 2005.

[21] F. P. Kelly, A. Maulloo, and D. Tan, "Rate control for communication networks: Shadow prices, proportional fairness, and stability," vol. 49, pp. 237-252, 1998.

[22] D. Tse and P. Viswanath, Fundamentals of Wireless Communication. Cambridge, UK: Cambridge University Press, 2005.

[23] T. Bu, L. Li, and R. Ramjee, "Generalized proportional fair scheduling in third generation wireless data networks," in Proc. IEEE Int. Conf. on Comp. Commu., Apr. 2006, pp. 1-12.

[24] S. K. Joshi, K. B. S. Manosha, M. Codreanu, and M. Latva-aho, "Dynamic inter-operator spectrum sharing via lyapunov optimization," IEEE Trans. Wireless Commun., submitted, 2016. 